Volume 70, Number 1, Pages 510-521(2021)

DOI: $10.31801 /$ cfsuasmas.724634

ISSN 1303-5991 E-ISSN 2618-6470

Received by the editors: April 21, 2020; Accepted: February 1, 2021

\title{
DIFFERENTIAL GEOMETRIC ASPECTS OF NONLINEAR SCHRÖDINGER EQUATION
}

\author{
Melek ERDOĞDU ${ }^{1}$ and Ayşe YAVUZ ${ }^{2}$ \\ ${ }^{1}$ Department of Mathematics - Computer, Necmettin Erbakan University, Konya, TURKEY \\ ${ }^{2}$ Department of Mathematics and Science Education, Necmettin Erbakan University, \\ Konya, TURKEY
}

\begin{abstract}
The main scope of this paper is to examine the smoke ring (or vortex filament) equation which can be viewed as a dynamical system on the space curve in $\mathbb{E}^{3}$. The differential geometric properties the soliton surface associated with Nonlinear Schrödinger (NLS) equation, which is called NLS surface or Hasimoto surface, are investigated by using Darboux frame. Moreover, Gaussian and mean curvature of Hasimoto surface are found in terms of Darboux curvatures $k_{n}, k_{g}$ and $\tau_{g}$. Then, we give a different proof of that the $s$-parameter curves of NLS surface are the geodesics of the soliton surface. As applications we examine two NLS surfaces with Darboux Frame.
\end{abstract}

\section{INTRODUCTION}

Investigation of motion of a vortex filament provides the crucial problems of mathematical physics and differential geometry. The work of Hasimoto in 1972 is one of the leading studies that guide us in solving these extremely important problems. It was concerned with an approximation to the self-induced motion of a thin isolated vortex filament traveling without stretching in an incompressible fluid. If the position vector of vortex filament is denoted by $r=r(s, t)$, then the equation

$$
r_{t}=r_{s} \times r_{s s}
$$

is hold 7. This equation is called the smoke ring or vortex filament equation. It can be considered that these vortex motions, which involve no change of form, correspond to traveling wave solutions of the Nonlinear Schrödinger (NLS) equation, 14. These kind of soliton surfaces that are associated with the NLS equation are called NLS surfaces or Hasimoto surfaces. Hasimoto stated the complex function

2020 Mathematics Subject Classification. 14J25, 53Z05.

Keywords and phrases. Smoke ring equation, vortex filament equation, NLS surface, darboux frame.

\ayasar@erbakan.edu.tr-Corresponding author; merdogdu@erbakan.edu.tr

(D) 0000-0002-0469-3786; 0000-0001-9610-6229.

(C)2021 Ankara University Communications Faculty of Sciences University of Ankara-Series A1 Mathematics and Statistics 
$q=\kappa e^{i \int \tau d s}$ of the curvature $\kappa$ and torsion $\tau$ of a space curve and obtained that if the curve evolves according to the vortex filament equation, then it gives the solution of the focussing cubic NLS equation

$$
i q_{t}+q_{s s}+v q|q|^{2}=0
$$

In the work [15], the binormal motion of curves of constant curvature is shown to lead to integrable extensions of the Dym equations. Moreover, the binormal motion of curves of constant torsion is shown to lead to integrable extensions of classical sine-Gordon equations. A reciprocal invariance is used to establish the existence of novel dual-soliton surfaces associated with a given soliton surface in the case of the extended Dym equation. Then a cc-ideal formulation is adduced to obtain a matrix Darboux invariance for the extended Dym and reciprocally linked $m^{2} K d V$ equations. At the same time, a Bäcklund transformation is introduced which allows us to generate the associated soliton surfaces. Similarities of both Bäcklund's and Bianchi's classical transformations are derived for the extended sine-Gordon system. In the study [13, NLS equation is examined in a general intrinsic geometric setting as introduced earlier in a kinematic analysis of certain hydrodynamic motions by [16], and subsequently applied in magneto-hydrodynamics by [17]. Furthermore, differential geometric properties of the soliton surfaces associated with NLS equation are obtained. Furthermore, the connection between Hasimoto derivation is stated. An intrinsic decomposition for the auto-Backlund transformation is obtained at level of the soliton surfaces. Finally, the connection between decomposition and a linear representation for NLS equation is investigated.

Emerging problems revealed that studies should be conducted for non-Euclidean geometries. The NLS equation of repulsive type for timelike curves and nonlinear heat system were examined in a general intrinsic geometric setting including a normal congruence in 3-dimensional Minkowski space in the study 5. Additionally, the motion of timelike surfaces correspond to the repulsive type NLS equation in timelike geodesic coordinates was studied in 6].

Erdoğdu and Özdemir investigated the Hasimoto surfaces in Minkowski 3-space and obtained the geometric properties of these surfaces 22. Kelleci and others examined the curvatures of Hasimoto surface according to Bishop frame and give some characterization of parameter curves of these surfaces [10]. Grbović and Nešović investigated vortex filament equation for pseudo null curves in Minkowski 3-space by using Bäcklund transformation in Minkowski 3-space [4].

In this paper, we investigate differential geometric properties of the soliton surface associated with Nonlinear Schrödinger (NLS) equation, which is called NLS surface or Hasimoto surface, are investigated by using the Darboux frame. Firstly, a brief summary is presented to provide the necessary background. We give a proof of that the $s$ parameter curves of NLS surface are the geodesics of the soliton surface and that this surface provides the NLS equation. Then, we discuss the geometric properties of NLS surface. We find Gaussian and mean curvature of NLS surface. 
Also, we obtain new results and the necessary and sufficient conditions for NLS surfaces to be flat or minimal surfaces. Finally, we investigate two different NLS surfaces as applications.

\section{Preliminaries}

In this section, we give the necessary information about the regular curves on surfaces to understand the main subject of the study. Let $\alpha: I \rightarrow M$ be a regular unit speed curve on the orientiable surface $M$. We may define Frenet frame $\{T, N, B\}$ at each points of the curve $\alpha$ where $T$ is unit tangent vector, $N$ is principal normal vector and $B$ is binormal vector. The Serret-Frenet formulas of the curve $\alpha$ are given by

$$
\begin{aligned}
& T^{\prime}(s)=\kappa(s) N(s), \\
& N^{\prime}(s)=-\kappa(s) T(s)+\tau(s) B(s), \\
& B^{\prime}(s)=-\tau(s) N(s),
\end{aligned}
$$

where the functions $\kappa$ and $\tau$ are called the curvature and the torsion of the curve $\alpha$, respectively. On the other hand, we may also define another orthonormal frame fields on the curve $\alpha$, which is called Darboux frame, since the curve $\alpha$ lies on the orientiable surface $M$. Darboux frame of $(\alpha, M)$ curve-surface couple includes unit tangent vector field $T$ of $\alpha$ and unit normal vector field of the surface $n=N \circ \alpha$ on the curve $\alpha$. To describe an orthonormal frame including these vector fields, there is only one way to choose last frame field as $g=n \times T$. This implies the following relations between Frenet and Darboux frames:

$$
\left[\begin{array}{l}
T \\
N \\
B
\end{array}\right]=\left[\begin{array}{ccc}
1 & 0 & 0 \\
0 & \cos \beta & -\sin \beta \\
0 & \sin \beta & \cos \beta
\end{array}\right]\left[\begin{array}{l}
T \\
n \\
g
\end{array}\right]
$$

and

$$
\left[\begin{array}{l}
T \\
n \\
g
\end{array}\right]=\left[\begin{array}{ccc}
1 & 0 & 0 \\
0 & \cos \beta & \sin \beta \\
0 & -\sin \beta & \cos \beta
\end{array}\right]\left[\begin{array}{l}
T \\
N \\
B
\end{array}\right],
$$

where $\beta$ is the angle between the vector fields $N$ and $n$. The derivative formulas of Darboux frame can be given as follows:

$$
\frac{d}{d s}\left[\begin{array}{c}
T \\
n \\
g
\end{array}\right]=\left[\begin{array}{ccc}
0 & k_{n} & k_{g} \\
-k_{n} & 0 & -t_{r} \\
-k_{g} & t_{r} & 0
\end{array}\right]\left[\begin{array}{c}
T \\
n \\
g
\end{array}\right],
$$

where

$$
\begin{aligned}
& k_{n}(s)=\kappa(s) \cos \beta(s), \\
& k_{g}(s)=-\kappa(s) \sin \beta(s), \\
& t_{r}(s)=-\beta^{\prime}(s)-\tau(s) .
\end{aligned}
$$


The functions $k_{g}$ is called geodesic curvature, $k_{n}$ is called normal curvature and $t_{r}$ is called geodesic torsion of $\alpha$. For a curve $\alpha(s)$ lying on a surface, the following cases are satisfied:

$\alpha(s)$ is a geodesic curve if and only if $k_{g}=0$,

$\alpha(s)$ is an asymptotic curve if and only if $k_{n}=0$,

$\alpha(s)$ is a principal line curve if and only if $t_{r}=0$.

The unit normal vector field $N$ on a surface $r$ can be defined by

$$
N=\frac{r_{s} \times r_{t}}{\left\|r_{s} \times r_{t}\right\|}
$$

The first fundamental form characterizes the interior geometry of the surface in a neighborhood of a given point. This means that measurements on the surface can be carried out by means of it. The first fundamental form is given by

$$
I=E d s^{2}+2 F d s d v+G d v^{2} .
$$

At the same time, the second fundamental form

$$
I I=e d s^{2}+2 f d s d v+g d v^{2},
$$

where

$$
e=\left\langle M_{s s}, N\right\rangle, \quad f=\left\langle M_{s v}, N\right\rangle \quad g=\left\langle M_{v v}, N\right\rangle .
$$

Gaussian curvature is given as

$$
K=\operatorname{det} S
$$

and the mean curvature is related to the trace as follows

$$
H=\frac{1}{2} \operatorname{tr} S
$$

Gaussian and mean curvatures of a surface are given by

$$
\begin{aligned}
& K=\frac{\operatorname{det} I I}{\operatorname{det} I}=\frac{e g-f^{2}}{E G-F^{2}}, \\
& H=\frac{E g+G e-2 F f}{2\left(E G-F^{2}\right)},
\end{aligned}
$$

respectively.

\section{Nonlinear Schrödinger Surfaces}

In this section, NLS surface or Hasimoto surface are investigated by using the Darboux frame and discuss the geometric properties of NLS surface. We find the Gaussian and mean curvatures of this surface. Also, we obtain some of the results and some necessary conditions for surfaces to be flat or minimal surfaces. We give a proof of that $s$-parameter curves of NLS surface are geodesics of the soliton surface and that this surface provides the NLS equation. 
The movement of a thin vortex in a thin vicous fluid by the motion of a curve propagating in $\mathbb{E}^{3}$ is described by the following equation:

$$
r_{t}=r_{s} \times r_{s s} \text {. }
$$

This is called the vortex filament or smoke ring equation, and can be viewed as a dynamically system on the space of curves.

Theorem 1. Suppose $r=r(s, t)$ is a NLS surface such that $r=r(s, t)$ is a unit speed curve with normal vector field for all $t$. Then the following is satisfied:

$$
\left[\begin{array}{l}
T \\
n \\
g
\end{array}\right]_{t}=\left[\begin{array}{ccc}
0 & \alpha & \lambda \\
-\alpha & 0 & -\gamma \\
-\lambda & \gamma & 0
\end{array}\right]\left[\begin{array}{l}
T \\
n \\
g
\end{array}\right],
$$

where $\{T, n, g\}$ is the Darboux frame, $\alpha, \lambda, \gamma$ are smooth functions given by following equalities

$$
\begin{aligned}
\alpha & =k_{g_{s}}-k_{n} t_{r}, \\
\lambda & =-k_{n_{s}}-k_{g} t_{r}, \\
\gamma & =\frac{1}{k_{g}^{2}+k_{n}^{2}}\left\{\begin{array}{c}
\left(k_{g} k_{g_{s}}+k_{n} k_{n_{s}}\right)_{s}-\left(k_{n_{s}}+k_{g_{s}} t_{r}\right)^{2} \\
-\left(k_{g_{s}}-k_{n} t_{r}\right)^{2}+k_{g_{t}} k_{n}-k_{n_{t}} k_{g}
\end{array}\right\} .
\end{aligned}
$$

Proof. We need to find these functions in terms of the curvatures $k_{g}, k_{n}, t_{r}$ of the solution curve $r=r(s, t)$ of smoke ring equation for all $t$. Using compatibility conditions $t_{t s}=t_{s t}$, we get

$$
\begin{aligned}
& \alpha_{s}=k_{n_{t}}+\gamma k_{g}-\lambda t_{r}, \\
& \lambda_{s}=k_{g_{t}}-\gamma k_{n}+\alpha t_{r},
\end{aligned}
$$

and using equality of $n_{t s}=n_{s t}$, we have

$$
\begin{aligned}
\alpha_{s} & =k_{n_{t}}+\gamma k_{g}-\lambda t_{r}, \\
\gamma_{s} & =\lambda k_{n}-\alpha k_{g}+t_{r_{t}} .
\end{aligned}
$$

Similarly, using equality of $g_{t s}=g_{s t}$, we get following equalities

$$
\begin{aligned}
& \gamma_{s}=\lambda k_{n}-\alpha k_{g}+t_{r_{t}}, \\
& \lambda_{s}=k_{g_{t}}-\gamma k_{n}+\alpha t_{r} .
\end{aligned}
$$

Thus, by the above relations, the following equations are obtained

$$
\begin{aligned}
& \alpha_{s}=k_{n_{t}}+\gamma k_{g}-\lambda t_{r}, \\
& \gamma_{s}=\lambda k_{n}-\alpha k_{g}+t_{r_{t}}, \\
& \lambda_{s}=k_{g_{t}}-\gamma k_{n}+\alpha t_{r} .
\end{aligned}
$$

Again by compatibility condition of $r_{s t}=r_{t s}$, we find the following equalities

$$
\begin{aligned}
\alpha & =k_{g_{s}}-k_{n} t_{r}, \\
\lambda & =-k_{n_{s}}-t_{r} k_{g} .
\end{aligned}
$$


Now, we assume that the velocity of the curve is of the form

$$
\gamma=\frac{1}{k_{g}^{2}+k_{n}^{2}}\left\{\begin{array}{c}
\left(k_{g} k_{g_{s}}+k_{n} k_{n_{s}}\right)_{s}-\left(k_{n_{s}}+k_{g_{s}} t_{r}\right)^{2} \\
-\left(k_{g_{s}}-k_{n} t_{r}\right)^{2}+k_{g_{t}} k_{n}-k_{n_{t}} k_{g}
\end{array}\right\} \text {. }
$$

For a solution of smoke ring equation, the velocity vector is given by

$$
r_{t}=r_{s} \times r_{s s}=t \times\left(k_{n} n+k_{g} g\right)=k_{g} n-k_{n} g .
$$

Theorem 2. If $r=r(s, t)$ is a NLS surface where $r=r(s, t)$ is a unit speed curve with normal vector field for all $t$, then the Gaussian curvature $K$ and mean curvature $H$ of $r=r(s, t)$ are

$$
\begin{aligned}
& K=\frac{1}{k_{g}^{2}+k_{n}^{2}}\left\{-\left(k_{g}^{2}+k_{n}^{2}\right) \gamma+k_{g_{t}} k_{n}-k_{n_{t}} k_{g}-\frac{\left(\alpha k_{n}+\lambda k_{g}\right)}{k_{g}^{2}+k_{n}^{2}}\right\}, \\
& H=\frac{1}{2\left(k_{g}^{2}+k_{n}^{2}\right) \sqrt{k_{g}^{2}+k_{n}^{2}}}\left\{\left(k_{g}^{2}+k_{n}^{2}\right) \gamma-k_{g_{t}} k_{n}+k_{n_{t}} k_{g}-\left(k_{g}^{2}+k_{n}^{2}\right)^{2}\right\},
\end{aligned}
$$

respectively. Here, $\kappa$ is the curvature function, and $\tau$ is the torsion function of the curve $r=r(s, t)$ for all $t$.

Proof. We have found the coefficients of first fundamental forms of the $r(s, t)$ as $E=1, F=0$, and $G=k_{g}^{2}+k_{n}^{2}$. Thus, we say that $E G-F^{2}=k_{g}^{2}+k_{n}^{2}$.

Normal vector field of the NLS surface is given by

$$
\begin{aligned}
N & =\frac{r_{s} \times r_{t}}{\left\|r_{s} \times r_{t}\right\|}=\frac{T \times\left(k_{g} n-k_{n} g\right)}{\left\|T \times\left(k_{g} n-k_{n} g\right)\right\|} \\
& =\frac{-k_{g} g-k_{n} n}{\sqrt{k_{g}^{2}+k_{n}^{2}}} .
\end{aligned}
$$

After some computations, one can easily obtain the coefficients of the second fundamental form as

$$
\begin{aligned}
& e=\frac{-\left(k_{g}^{2}+k_{n}^{2}\right)}{\sqrt{k_{g}^{2}+k_{n}^{2}}}, \\
& f=\frac{t_{r}\left(k_{g}^{2}+k_{n}^{2}\right)-k_{n} k_{g_{s}}+k_{g} k_{n_{s}}}{\sqrt{k_{g}^{2}+k_{n}^{2}}}, \\
& g=\frac{1}{\sqrt{k_{g}^{2}+k_{n}^{2}}}\left\{\left(k_{g} k_{g_{s}}+k_{n} k_{n_{s}}\right)_{s}-\left(k_{n_{s}}+k_{g} t_{r}\right)^{2}-\left(k_{g_{s}}-t_{r} k_{n}\right)^{2}\right\} .
\end{aligned}
$$

Thus, the Gaussian curvature $K$ and mean curvature $H$ of the surface $r(s, t)$ are

$$
K=\frac{1}{k_{g}^{2}+k_{n}^{2}}\left\{-\left(k_{g}^{2}+k_{n}^{2}\right) \gamma+k_{g_{t}} k_{n}-k_{n_{t}} k_{g}-\frac{\left(\alpha k_{n}+\lambda k_{g}\right)}{k_{g}^{2}+k_{n}^{2}}\right\},
$$




$$
H=\frac{1}{2\left(k_{g}^{2}+k_{n}^{2}\right) \sqrt{k_{g}^{2}+k_{n}^{2}}}\left\{\left(k_{g}^{2}+k_{n}^{2}\right) \gamma-k_{g_{t}} k_{n}+k_{n_{t}} k_{g}-\left(k_{g}^{2}+k_{n}^{2}\right)^{2}\right\},
$$

respectively.

Theorem 3. Suppose $r=r(s, t)$ is a $N L S$ surface in $\mathbb{E}^{3}$, the s-parameter curves of the surface $r=r(s, t)$ are geodesics.

Proof. Suppose $r=r(s, t)$ is a NLS surface such that $r=r(s, t)$ is unit speed curve for all $t$. We know that

$$
r_{s s}=k_{n} n+k_{g} g \text {. }
$$

And the surface normal of $r=r(s, t)$ is

$$
N=\frac{r_{s} \times r_{t}}{\left\|r_{s} \times r_{t}\right\|}=\frac{-k_{g} g-k_{n} n}{\sqrt{k_{g}^{2}+k_{n}^{2}}} .
$$

Thus, $r_{s s}$ is parallel to surface normal which means $s$-parameter curves of the surface are geodesics.

Remark 4. Since the s-parameter curves of NLS surface are geodesics, this implies that $k_{g}=0$.

Theorem 5. Suppose $r=r(s, t)$ is a $N L S$ surface in $\mathbb{E}^{3}$. $t$-parameter curves of the surface $r=r(s, t)$ are geodesics if and only if $k_{n_{s}}=0$.

Proof. By above remark, we have

$$
r_{t}=k_{g} n-k_{n} g=-k_{n} g
$$

Thus, we obtain the tangent vector field of $t$-parameter curve as

$$
\bar{t}=\frac{r_{t}}{\left\|r_{t}\right\|}=-g .
$$

Then, the third vector field of Darboux frame of $t$-parameter curve is found

$$
\bar{g}=-n \times \bar{t}=-n \times(-g)=-T .
$$

The geodesic curvature of the $t$-parameter curve is obtained as

$$
\overline{k_{g}}=\left\langle\bar{t}_{t}, \bar{g}\right\rangle=\langle\lambda T-\gamma n,-T\rangle=\lambda
$$

where

$$
\lambda=-k_{n_{s}}-k_{g} t_{r}=-k_{n_{s}} .
$$

Therefore, $t$-parameter curves of the surface $r=r(s, t)$ are geodesics if and only if $k_{n_{s}}=0$.

Theorem 6. Suppose $r=r(s, t)$ is a $N L S$ surface in $\mathbb{E}^{3}, N L S$ equation

$$
i q_{t}+q_{s s}+v q|q|^{2}=0
$$

is provide with Hasimoto transformation $q= \pm k_{n} e^{i \int-t_{r} d s}$. 
Proof. We know that Hasimoto transformation with Frenet frame is obtained as follows

$$
q=\kappa e^{i \sigma}
$$

such that $\sigma=\int \tau d s$. Since the following equations are satisfied

$$
\begin{aligned}
\kappa^{2} & =k_{n}^{2}+k_{g}^{2}, \\
t_{r}(s) & =-\beta^{\prime}(s)-\tau(s),
\end{aligned}
$$

and $k_{g}=0$, we obtain that Hasimoto transformation with Darboux frame as follows

$$
q= \pm k_{n} e^{i \int-t_{r} d s} .
$$

By taking derivative of $q$ according to $t$ parameter, we get

$$
q_{t}=\left(k_{n_{t}}+i k_{n} t_{r_{t}}\right) e^{i \int-t_{r} d s} .
$$

We also know that

$$
k_{n_{t}}=\alpha_{s}+\lambda t_{r}, t_{r_{t}}=\gamma_{s}-\lambda k_{n}
$$

such that

$$
\begin{aligned}
\alpha & =-k_{n} t_{r}, \\
\lambda & =-k_{n_{s}}, \\
\gamma & =\frac{1}{k_{n}}\left(k_{n_{s s}-} k_{n} t_{r}^{2}\right),
\end{aligned}
$$

by equations (5) and (6). Therefore, we obtain

$$
\begin{aligned}
k_{n_{t}} & =\left(-k_{n} t_{r}\right)_{s}-k_{n_{s}} t_{r} \\
& =-2 k_{n_{s}} t_{r}-k_{n} t_{r_{s}}
\end{aligned}
$$

and

$$
\begin{aligned}
t_{r_{t}} & =\left(\frac{1}{k_{n}}\left(k_{n_{s s}}-k_{n} t_{r}^{2}\right)\right)_{s}+k_{n_{s}} k_{n} \\
& =\left(\frac{k_{n_{s s}}}{k_{n}}-t_{r}^{2}+\frac{k_{n}^{2}}{2}\right)_{s} .
\end{aligned}
$$

If we substitute above equations into Equation (3.8), then we obtain

$$
q_{t}=\left(-2 k_{n_{s}} t_{r}-k_{n} t_{r_{s}}+i k_{n}\left(\frac{k_{n_{s s}}}{k_{n}}-t_{r}^{2}+\frac{k_{n}^{2}}{2}\right)\right) e^{i \int-t_{r} d s}
$$

Thus, we also get

$$
q_{s s}=\left(k_{n_{s s}}+2 i k_{n_{s}} t_{r}+i k_{n} t_{r_{s}}-k_{n} t_{r}^{2}\right) e^{i \int-t_{r} d s} .
$$

According to above findings, it is seen that the following NLS equation is satisfied

$$
i q_{t}+q_{s s}+v q|q|^{2}=0 .
$$


Remark 7. The vortex filament equation can be rewritten in terms of Darboux frame as follows

$$
r_{t}=k_{g} n-k_{n} g=-k_{n} g
$$

such that

$$
\begin{aligned}
& n=\cos \left(\int-t_{r} d s\right) N+\sin \left(\int-t_{r} d s\right) B, \\
& g=-\sin \left(\int-t_{r} d s\right) N+\cos \left(\int-t_{r} d s\right) B .
\end{aligned}
$$

Corollary 8. If $r=r(s, t)$ is a NLS surface where $r=r(s, t)$ is a unit speed curve with normal vector field for all $t$, then the Gaussian curvature $K$ and mean curvature $H$ of $r=r(s, t)$ are given by

$$
\begin{aligned}
& K=-\gamma-\frac{\alpha}{k_{n}^{3}}, \\
& H=\frac{\gamma}{2 k_{n}}-\frac{1}{2 k_{n}^{3}},
\end{aligned}
$$

respectively, such that

$$
\begin{gathered}
\alpha=k_{n} t_{r} \\
\lambda=-k_{n_{s}} \\
\gamma=\frac{1}{k_{n}^{2}}\left\{\left(k_{n} k_{n_{s}}\right)_{s}-\left(k_{n_{s}}\right)^{2}-\left(-k_{n} t_{r}\right)^{2}\right\} .
\end{gathered}
$$

Proof. Since $s$-parameter curves of Hasimoto surface $r=r(s, t)$ are geodesics, then it should be $k_{g}=0$. This implies that the Gaussian curvature $K$ and mean curvature $H$ of $r=r(s, t)$ are obtained as

$$
\begin{aligned}
& K=-\gamma-\frac{\alpha}{k_{n}^{3}}, \\
& H=\frac{\gamma}{2 k_{n}}-\frac{1}{2 k_{n}^{3}}
\end{aligned}
$$

by Theorem 2 .

Corollary 9. NLS surface $r=r(s, t)$ is developable if and only if

$$
\left(k_{n} k_{n_{s}}\right)_{s}=k_{n_{s}}^{2}+k_{n}^{2} t_{r}^{2}-t_{r} .
$$

Corollary 10. NLS surface $r=r(s, t)$ is minimal surface if and only if

$$
\left(k_{n} k_{n_{s}}\right)_{s}=1+k_{n_{s}}^{2}+k_{n}^{2} t_{r}^{2} .
$$




\section{Applications}

Example 11. Consider the NLS surface with the following parametrization

$$
r(s, t)=\left(s-\frac{2}{3} \tanh (3 s),-\frac{2}{3} \operatorname{sech}(3 s) \cos (9 t),-\frac{2}{3} \operatorname{sech}(3 s) \sin (9 t)\right) .
$$

Let us examine Darboux frame of the curves $r=r(s, t)$ for all $t \in \mathbb{R}$ and $N L S$ surface. We find Darboux vector fields as follows:

$T(s, t)=\left(2 \tanh ^{2}(3 s)-1,2 \operatorname{sech}(3 s) \tanh (3 s) \cos (9 t), 2 \operatorname{sech}(3 s) \tanh (3 s) \sin (9 t)\right)$, $n(s, t)=\left(-2 \tanh (3 s) \operatorname{sech}(3 s),\left(\tanh ^{2}(3 s)-\operatorname{sech}^{2}(3 s)\right) \cos (9 t)\right.$,

$$
\left.\left(\tanh ^{2}(3 s)-\operatorname{sech}^{2}(3 s)\right) \sin (9 t)\right)
$$

$g(s, t)=(0, \sin (9 t),-\cos (9 t))$.

Then we obtain Darboux curvatures as follows

$$
\begin{aligned}
k_{n}(s, t) & =-6 \operatorname{sech}(3 s), \\
k_{g}(s, t) & =0, \\
t_{r}(s, t) & =0 .
\end{aligned}
$$

Furthermore, we get the Gaussian and mean curvatures of the NLS surface

$$
\begin{aligned}
& K(s, t)=-9\left(\tanh ^{2}(3 s)-\operatorname{sech}^{2}(3 s)\right), \\
& H(s, t)=-\frac{9}{2} \operatorname{sech}(3 s)+\frac{3}{4 \operatorname{sech}(3 s)} .
\end{aligned}
$$

The following figure illustrate NLS surface and its geodesic some of s-parameter curves on the surface.

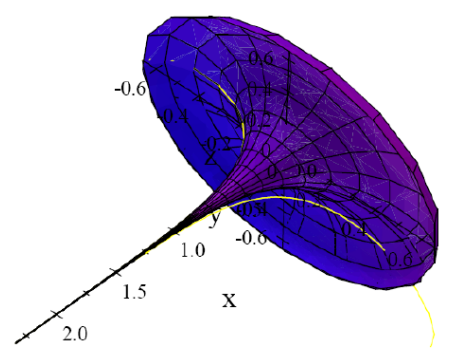

Figure 1: Two geodesics on NLS surface are illustrated by the color yellow

Example 12. Let $r(s, t)$ be a NLS surface with following parametric expression

$$
r(s, t)=(\cos s, \sin s, t) .
$$


We find the Darboux frame of the curve as follows

$$
\begin{aligned}
T(s) & =(-\sin s, \cos s, 0), \\
n(s) & =(\cos s, \sin s, 0), \\
g(s) & =(0,0,1),
\end{aligned}
$$

and we find geodesic curvature, normal curvature, and geodesic torsion of $\alpha$ as follows

$$
\begin{aligned}
& k_{n}=-1, \\
& k_{g}=t_{r}=0 .
\end{aligned}
$$

The following figure illustrate NLS surface and its geodesic some of s-parameter curve on the surface

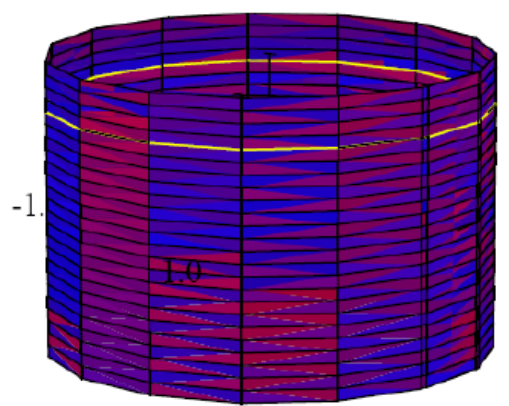

Figure 2: The geodesic on ruled NLS surface is illustrated by the color yellow

Authors Contribution Statement The authors confirm contribution to the paper as follows: M. Erdoğdu and A. Yavuz conceived of the presented idea. Both authors developed the theory and performed the computations. All authors reviewed the results and approved the final version of the manuscript.

Declaration of Competing Interests The author declares no conflicts of interest in this paper.

\section{REFERENCES}

[1] Ding, Q., Inoguchi, J., Schrödinger flows, binormal motion for curves and second AKNShierarchies, Chaos Solitons and Fractals, 21 (3) (2004), 669-677. https://doi.org/10.1016/ j.chaos.2003.12.092

[2] Erdoğdu, M., Özdemir, M., Geometry of Hasimoto surfaces in Minkowski 3-space, Math. Phys. Anal. Geom., 17 (1) (2014), 169-181. DOI:10.1007/s11040-014-9148-3

[3] Fujika, A., Inoguchi, J., Spacelike surfaces with harmonic inverse mean curvature, J. Math. Sci. Univ. Tokyo, 7 (4) (2000), 657-698. 
[4] Grbovic, M. and Nesovic, E., On Bäcklund transformation and vortex filament equation for pseudo null curves in Minkowski 3-space, Int. J. Geom. Methods Mod. Phys. 13 (6) (2016), 1-14. https://doi.org/10.1142/S0219887816500778

[5] Gürbüz, N., Intrinstic geometry of NLS equation and heat system in 3-dimensional minkowski space, Adv. Stud. Theor., 4 (1) (2010), 557-564.

[6] Gürbüz, N., The motion of timelike surfaces in timelike geodesic coordinates, Int. J. Math. Anal., 4 (2010), 349-356.

[7] Hasimoto, H., A soliton on a vortex filament, J. Fluid. Mech., 51 (3) (1972), 477-485. https: //doi.org/10.1017/S0022112072002307

[8] Inoguchi, J., Timelike surfaces of constant mean curvature in Minkowski 3-space, Tokyo J. Math., 21 (1) (1998), 141-152 DOI:10.3836/tjm/1270041992

[9] Inoguchi, J., Biharmonic curves in Minkowski 3-space, Int. J. Math. Math. Sci., (2003), 1365-1368. https://doi.org/10.1155/S016117120320805X

[10] Kelleci, A., Bektaş, M., Ergüt, M., The Hasimoto surface according to bishop frame, Adıyaman University Journal of Science, 9 (2019), 13-22.

[11] Özdemir, M., Ergin, A.A., Rotations with unit timelike quaternions in Minkowski 3-space, $J$. Geom. Phys., 56 (2) (2006), 322-336. https://doi.org/10.1016/j.geomphys.2005.02.004

[12] Özdemir, M., Ergin, A.A., Parallel frames of non-lightlike curves, Missouri Journal of Mathematical Sciences, 20 (2) (2008), 127-137. DOI:10.35834/mjms/1316032813

[13] Rogers, C., Schief, W.K., Intrinsic geometry of the NLS equation and its backlund transformation, Stud. Appl. Math., 101 (3) (1998), 267-288. https://doi.org/10.1111/1467-9590. 00093

[14] Rogers, C., Schief, W.K., Backlund and Darboux Transformations: Geometry of Modern Applications in Soliton Theory, Cambridge University Press, 2002. https://doi.org/10. 1017/CB09780511606359

[15] Schief, W.K., Rogers, C., Binormal motion of curves of constant curvature and torsion, generation of soliton surfaces, Proc. R. Soc. Lond. A., 455 (1988) (1999), 3163-3188. https: //doi.org/10.1098/rspa.1999.0445

[16] Marris, A. W., Passman, S. L., Vector fields and flows on developable surfaces, Arch. Ration. Mech. Anal., 32 (1) (1969), 29-86. https://doi.org/10.1007/BF00253256

[17] Rogers, C., Kingston, J. G. Nondissipative magneto-hydrodynamic flows with magnetic and velocity field lines orthogonal geodesics, SIAM J. Appl. Math., 26 (1) (1974), 183-195. https: //doi.org/10.1137/0126015 